\title{
A Wavelet based Digital Watermarking Method using Thresholds on Intermediate Bit Values
}

\author{
S.MaruthuPerumal \\ Research Scholar Dr MGR University Chennai, \\ Associate Professor \\ Godavari Institute of Engineering and Technology \\ Rajahmundry, A.P. India
}

\author{
Dr.V.VijayaKumar \\ Professor \& Dean Department of Computer Sciences, \\ Head Srinivasa Ramanujan Research Forum (SRRF) \\ Godavari Institute of Engineering and Technology \\ Rajahmundry, A.P. India
}

\begin{abstract}
Many researchers are concentrating to provide a high confidentiality and data integrity to protect against unauthorized access. The network security is becoming a very important and crucial aspect as the number of data being exchanged and number of users being communicated on the internet increases rapidly day by day. Therefore confidentiality of users and data integrity are required to protect against unauthorized access. To address the issues of authentication, the present paper proposes a novel wavelet based Digital Watermarking (DW) approach with two steps. In the first step a Threshold, based on Intermediate Bit Values (TIBV) of image pixels is proposed by selecting the image pixel for inserting the watermark. In the second step Alattar's method is used for inserting the Digital Watermark (DW) bits in the selected pixels of the first step. These two steps make the proposed TIBV-DW method as more effective and difficult to break. To test the efficacy of the proposed TIBVDW method, various statistical measures by considering combinations of various attacks are evaluated on various experimental images, which indicate high robustness, imperceptibility, un-ambiguity, confidentiality and integrity of the present method.
\end{abstract}

\section{Keywords}

Wavelet Transformation, Threshold based Intermediate Bit Values (TIBV), Alattar's Method.

\section{INTRODUCTION}

With the development of computer, multimedia and network technologies, the amount of visual information available in digital format has grown exponentially recently. This has resulted in information explosion [9] and the great advancement in the field of internet, which has facilitated the transmission, wide distribution, and access of multimedia data in an effortless manner [2-5]. Digital watermarking techniques are used to provide copyright protection and authentications for digital images, audio and video data. The digital watermarking technique is proposed as a method to embed perceptible or imperceptible signal into multimedia data for claiming the ownership. That's why there is a lot of research in the field of digital watermarking. Each watermarking application has its own needs that determine the required attributes of the watermarking system and drive the choice of techniques used for embedding and detecting the watermark [6-8]. One of the methods that can authenticate the completeness of information and protect intellectual property rights and copyrights is the data embedding scheme. It can also be used to enrich the information content and enhance its value. But the data embedding process usually alters the original data. Unfortunately, even if the change is very slight, some kinds of data are rendered unworkable. Therefore, the reversible data embedding method is explored. The reversible data embedding method embeds the data into a cover media in a reversible fashion. From the embedded media, the data can be extracted and the original media can be recovered. Of course, the data can be the copyright or other information about the cover media. In the last decade, many reversible data embedding methods have been proposed $[30,31,32,33,34]$.

A high-capacity and low-distortion reversible data embedding scheme based on the difference expansion transform of a pair of pixels, divides the image in to pairs of pixels [29]. These pairs are not expected to cause an overflow or under flow. They are embedded one bit into the difference of the pixels of each pair. The location map indicating which pairs are embedded data is compressed and included in the payload. In a single pass, this algorithm [29] can embed 0.5bits/pixel at most. This method [29] is improved by considering the correlation between the target pixel and its surrounding pixels to determine the degree of the difference expansion for message data embedding [28]. This method [28] strengthened the embedding ability and improved the computation efficiency. In a single pass, this method [28] can embed 1bit/pixel at most. A method using a difference expansion of vectors, which is an extension of method [28], is proposed to increase the hiding ability. This method is known as Alattar's method. Alattar also used the generalized integer transform of vectors to improve the embedded image quality. In Alattar's method, k pixels can embed k-1 bits. However, like Tian's method [28], the location map and other extra information are needed to restore the original cover image, so the embedding ability of their method is as high as $(\mathrm{k}-1) / \mathrm{k}$ bits/pixel. Increasing the embedding capacity, maintaining the reversible characteristic and simultaneously decreasing the destruction of the original image are the technical challenges of the reversible data embedding problem. Our method not only takes care of the embedded digital watermarking quality but also provides high authentication in choosing the selected pixels for embedding the information.

The proposed method embeds more than one bit by using Alattar's method in the selected pixels based on TIBV approach in a single pass using wavelet decomposition and also derives high embedded image quality. The rest of this paper is organized as follows: In Section 1.1 we briefly review Wavelet transformation. Section 2 and 2.1 presents the proposed TIBV method and Alattar's method respectively in detail. Section 3 
presents the experimental results. Finally, conclusions are summarized in Section 4.

\subsection{Wavelet Transformation}

A wavelet is a kind of mathematical function used to divide a given function or continuous-time signal into components of different frequency and to study each component with a resolution that matches its scale. A wavelet transform is the representation of a function by wavelets. The wavelets are scaled and translated copies (known as "daughter wavelets") of a finite-length or fast-decaying oscillating waveforms (known as the "mother wavelet"). Today wavelets play a significant role in Astronomy, Acoustics, Nuclear Engineering, Sub band Coding, Signal and Image Processing, Neurophysiology, Music, Magnetic Resonance Imaging, Speech Discrimination, Optics, Turbulence, Earthquake Prediction, Radar, Computer and Human Vision, Data Mining and Pure Mathematics Applications such as Solving Partial Differential Equations etc.

In the wavelet transform [18],[19],[20],[21],[22],[23],[24],[25], [26],[27] an image signal can be analyzed by passing it through an analysis filter bank followed by a decimation operation. This analysis filter bank consists of a low pass and a high pass filter at each decomposition stage.

When the signal passes through these filters it splits into two bands. The low pass filter, which corresponds to an averaging operation, extracts the coarse information of a signal. The high pass filter, which corresponds to a differencing operation, extracts the detail information of the signal. The output of the filtering operations is then decimated by two [18], [24], [26].Because of decimation the total size of the transformed image is same as the original image, which is shown in figure 1. Then, it is followed by filtering the sub image along the y-dimension and decimated by two. Finally, the image splits into four bands denoted by low-low (LL1), high-low (HL1), low-high (LH1) and high-high (HH1) after one-level decomposition as depicted in figure 2. The sub bands labeled, LL1 HL1, LH1, and HH1 represent the finest scale wavelet coefficients. To obtain the next coarser scaled wavelet coefficients, the sub band LL1 is further decomposed and critically sub sampled. This process is repeated several times, which is determined by the application at hand. Furthermore, from these DWT coefficients, the original image can be reconstructed. This reconstruction process is called the inverse DWT (IDWT). If $\mathrm{I}[\mathrm{m}, \mathrm{n}]$ represents an image, the DWT and IDWT for $\mathrm{I}[\mathrm{m}, \mathrm{n}]$ can be similarly defined by implementing the DWT and IDWT on each dimension and separately. Figure 3 shows second level of filtering. This process of filtering the image is called 'Pyramidal decomposition' of image. Figure 4 shows the results of different levels of decomposition of Lena image.

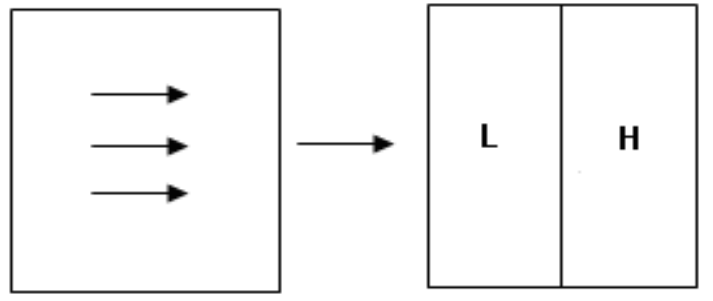

Figure 1: Horizontal Wavelet Transform.

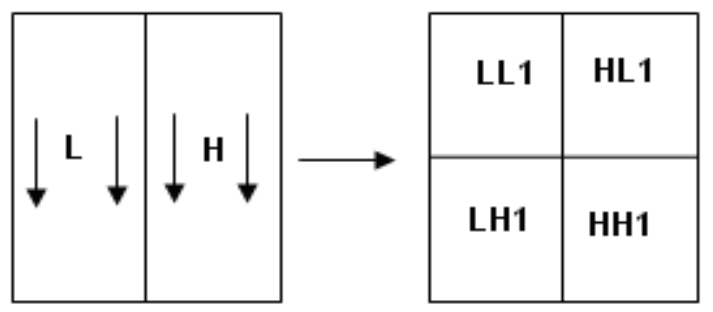

Figure 2: Vertical Wavelet Transform of Figure.1.

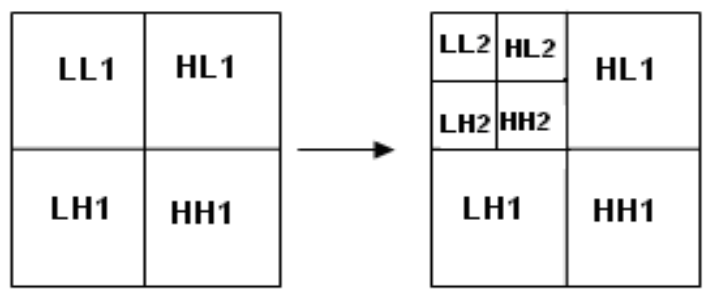

Figure 3: Second Level Wavelet Transform.

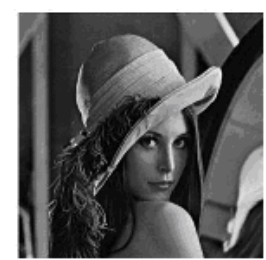

(a)

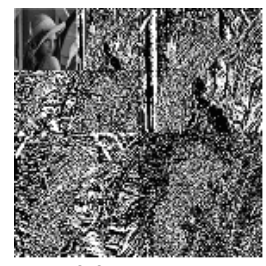

(c)

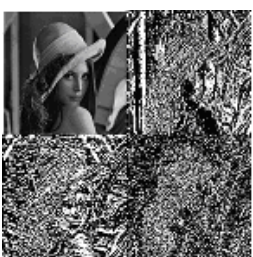

(b)

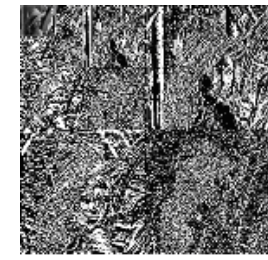

(d)
Figure 4: (a) Original Lena Image (b) Level-1 Wavelet Transformed Lena Image (c) Level-2 Wavelet Transformed Lena Image (d) Level-3 Wavelet Transformed Lena Image. 


\section{THRESHOLD BASED INTERMEDIATE BIT VALUES APPROACH OF DIGITAL WATERMARKING}

A digital image is composed of a set of two dimensional arrays of pixels values. Each pixel is represented by eight bits. The present paper considers the six Intermediate bit values of a pixel leaving the LSB and MSB of the pixel. In the proposed TIBVDW approach the digital water mark is embedded on the Selected pixels based on various thresholds on intermediate bit values. The digital watermark is embedded, based on Alattar's simplified version of difference expansion method. The novelty of the present method is, it uses three types of thresholds, for selecting pixels, based on the maximum number of occurrence value, $($ Min $+\max ) / 2$ and mean, for inserting the watermark. If two or more Intermediate Bit Values are having the same frequency of occurrence count, then the Intermediate Bit values having the highest value is chosen, in the case of the threshold based on the frequency of occurrence count of intermediate bit values, for employing the Alattar's method. On the selected pixels from top left to bottom right the Alattar's method is applied. To test the efficacy of the proposed TIBV-DW method, various statistical measures are evaluated on the different thresholds of the watermarked images. The Alattar's method [1] is briefly explained below.

\subsection{Alattar's Method of Watermark Insertion and Extraction}

Alattar's simplified version of difference expansion is used to increase the hiding ability. In Alattar's method, k pixels can embed k-1 bits, and the method is explained with an example as given below.

Consider the three vector pixels and two binary values of watermark data. Let the vector pixels be $(\mathrm{u} 1, \mathrm{u} 2, \mathrm{u} 3)=(94$, $92,91)$ and two binary values of watermark data be $b 1=1$, $\mathrm{b} 2=0$.

The weighted average $\mathrm{d} 1$ and the differences $\mathrm{d} 2, \mathrm{~d} 3$ are calculated by the following equation (1)

$\left.\begin{array}{l}d_{1}=\left\lfloor\frac{a_{1} u_{1}+a_{2} u_{2}+a_{3} u_{3}}{a_{1}+a_{2}+a_{3}}\right\rfloor=92 \\ d_{2}=u_{2}-u_{1}=-2 \\ d_{3}=u_{3}-u_{1}=-3\end{array}\right\}$

where $\mathrm{a} 1, \mathrm{a} 2$ and $\mathrm{a} 3$ are the predefined weights. In this example, we let $\mathrm{a} 1=\mathrm{a} 2=\mathrm{a} 3=1$.

Then the differences are expanded and the data bits are embedded into the new differences by using the following equation (2)

$\left.\begin{array}{ll}\tilde{d}_{1}=d_{1} & =92 \\ \tilde{d}_{2}=2 \times d_{2}+b_{1} & =-3 \\ \tilde{d}_{3}=2 \times d_{3}+b_{2} & =-6\end{array}\right\}$
The vector $\left(u_{1}^{\prime}, u_{2}^{\prime}, u_{3}^{\prime}\right)$ which is the final embedded pixel values is given in (3)

$$
\left.\begin{array}{l}
u_{1}^{\prime}=d_{1}-\left\lfloor\frac{a_{2} \times \tilde{d}_{2}+a_{3} \times \tilde{d}_{3}}{a_{1}+a_{2}+a_{3}}\right\rfloor=95 \\
u_{2}^{\prime}=\tilde{d}_{2}+d_{1}-\left\lfloor\frac{a_{2} \times \tilde{d}_{2}+a_{3} \times \tilde{d}_{3}}{a_{1}+a_{2}+a_{3}}\right\rfloor=92 \\
u_{3}^{\prime}=\tilde{d}_{3}+d_{1}-\left\lfloor\frac{a_{2} \times \tilde{d}_{2}+a_{3} \times \tilde{d}_{3}}{a_{1}+a_{2}+a_{3}}\right\rfloor=89
\end{array}\right\}
$$

Watermark will be extracted by using the Alattar's extraction phase as given below. The weighted average are calculated first as given in equation (4)

$$
\begin{aligned}
& \tilde{d}_{1}=\left\lfloor\frac{a_{1} \times u_{1}^{\prime}+a_{2} \times u_{2}^{\prime}+a_{3} \times u_{3}^{\prime}}{a_{1}+a_{2}+a_{3}}\right\rfloor=92 \\
& \tilde{d}_{2}=u_{2}^{\prime}-u_{1}^{\prime} \\
& \left.\begin{array}{l}
=-3 \\
=-6
\end{array}\right\}
\end{aligned}
$$

$$
\left.\begin{array}{l}
b_{1}=\tilde{d}_{2}-2 \times\left\lfloor\frac{\tilde{d}_{2}}{2}\right\rfloor \quad=1 \\
b_{2}=\tilde{d}_{3}-2 \times\left\lfloor\frac{\tilde{d}_{3}}{2}\right\rfloor
\end{array}\right\rfloor
$$

The original weighted average and the differences are calculated by equation (6)

$$
\begin{array}{ll}
d_{1}=\tilde{d}_{1} & =92 \\
d_{2}=\left\lfloor\frac{\tilde{d}_{2}}{2}\right\rfloor & =-2 \\
d_{3}=\left\lfloor\frac{\tilde{d}_{3}}{2}\right\rfloor & =-3
\end{array}
$$


Finally, the original pixel values are recovered by equation (7)

$$
\left.\begin{array}{ll}
u_{1}=d_{1}-\left\lfloor\frac{a_{2} \times d_{2}+a_{3} \times d_{3}}{a_{1}+a_{2}+a_{3}}\right\rfloor & =94 \\
u_{2}=d_{2}+u_{1} & =92 \\
u_{3}=d_{3}+u_{1} & =91
\end{array}\right\}
$$

\section{RESULTS AND DISCUSSIONS}

The present paper used various quality of measures of watermarked image like Mean Square Error (MSE), Root Mean Square Error (RMSE), Peak Signal to Noise Ratio (PSNR), Signal to Noise Ratio (SNR), Root Signal to Noise Ratio (RSNR) and Normalized Correlation Coefficient (NCC) given in Equations (8) to (13) to identify the quality of the watermarked image and reconstructed image. The embedding distortion performance is usually measured by the MSE and RMSE as given by the equation (8) and (9). A better perceptual quality of the image is acquired for lower values of MSE and RMSE. Signal to Noise Ratio (SNR) and Root Signal to Noise Ratio (RSNR) measures, estimates the quality of the reconstructed image compared with an original image, and they are defined in equation (10) and (11) respectively. A higher value of SNR and RSNR indicates the better quality of the reconstructed image. To study the quality of watermarked image, the peak signal to noise ratio PSNR is used. In general, a processed image is acceptable to the human eyes if its PSNR is greater than $30 \mathrm{~dB}$. The larger the PSNR, the better is the image quality. The PSNR is defined as given by the equation (12). To verify the robustness of any digital watermarking method, Normalized Cross Correlation (NCC) is used, which is defined in the equation (13). Using NCC, the similarity value nearer to 1 is considered as acceptable.

$M S E=\frac{1}{M \times N} \sum_{i=0}^{M-1} \sum_{j=0}^{N-1}(f(i, j)-g(i, j))^{2}$

$R M S E=\sqrt{\frac{1}{M \times N} \sum_{i=0}^{M-1} \sum_{j=0}^{N-1}(f(i, j)-g(i, j))^{2}}$

$$
S N R=\frac{\sum_{i=0}^{M-1} \sum_{j=0}^{N-1} g(i, j)^{2}}{\sum_{i=0}^{M-1} \sum_{j=0}^{N-1}(g(i, j)-f(i, j))^{2}}
$$

$$
\begin{aligned}
& R S N R=\sqrt{\frac{\sum_{i=0}^{M-1} \sum_{j=0}^{N-1} g(i, j)^{2}}{\sum_{i=0}^{M-1} \sum_{j=0}^{N-1}(g(i, j)-f(i, j))^{2}}} \\
& P S N R=10^{*} \log _{10}\left(\frac{255^{2}}{M S E}\right) \\
& N C C=\frac{\sum_{i=0}^{N-1} w(i) \times w^{*}(i)}{\sum_{i=0}^{N-1}(w(i))^{2}}
\end{aligned}
$$

where $f(i, j)$ and $g(i, j)$ are the original image and watermarked image with coordinate position $(i, j), w(i)$ and $w^{*}(i)$ are the original watermark text and the extracted watermark text.

For the experimental analysis on the proposed TIBV-DW method, with different thresholds, different images of different sizes are taken as shown in figure 5. The cover images considered in the proposed TIBV-DW method are Lena, Cameraman, Barbara, Circle, Pepper, Monalisa and Line images which are shown from figure $5(\mathrm{a})$ to $5(\mathrm{~g})$ respectively. The proposed TIBV-DW method is applied on 1-level decomposed images. In the wavelet based watermarked image, a set of 18 characters "srinivasaramanujan" is embedded as water mark. The above discussed quality parameters are estimated on the proposed TIBV-DW images with different thresholds that is max number of occurrence, $(\min +\max ) / 2$ and mean and are listed in the tables 1,2 and 3 respectively.

For testing the robustness of the proposed TIBV-DW method Gaussian Filtering, Salt and Pepper Noise, Rotation and Cropping attacks are used combinedly on the watermarked images and the performance is tabulated in the table 4 . That is all the attacks are applied on the watermarked image instead of one or two.

The figure 6(a) to $6(\mathrm{~g})$ shows the one level Haar wavelet decomposed images. The figure 7(a) to7(g) shows the images with the watermark text "srinivasaramanujan" embedded and the figure $8(\mathrm{a})$ to $8(\mathrm{~g})$ shows the images after extraction of watermark.

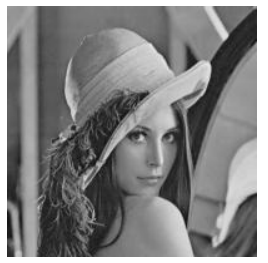

(a)

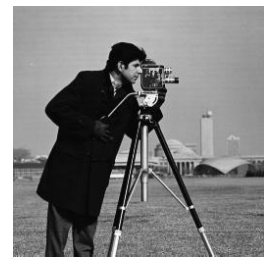

(b)

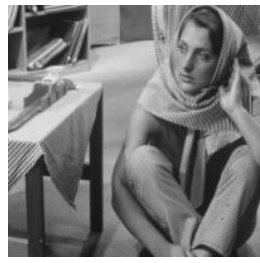

(c) 


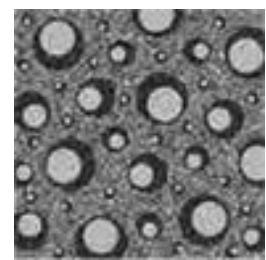

(d)

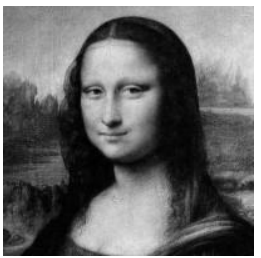

(e)

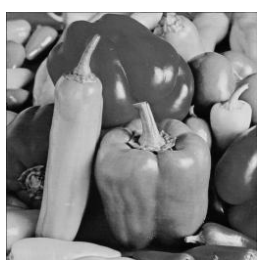

(f)

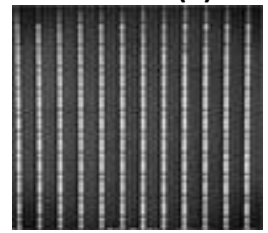

(g)

Figure 5: Original Images (a) Lena Image200×200 (b) Cameraman Image 256x256 (c) Barbara Image 512x512 (d) Circle Image256x256 (e) Monalisa Image200x200 (f) Pepper Image 256x256 (g) Line Image 256x256

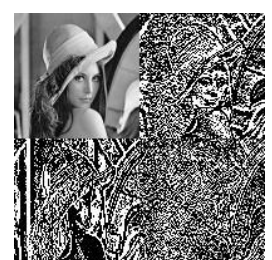

(a)

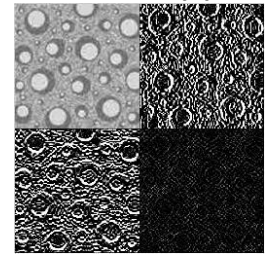

(d)

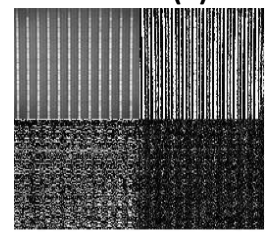

(g)

Figure 6: One level Decomposed Image (a) Lena Image (b) Cameraman Image (c) Barbara Image (d) Circle Image (e) Monalisa Image (f) Pepper Image (g) Line Image

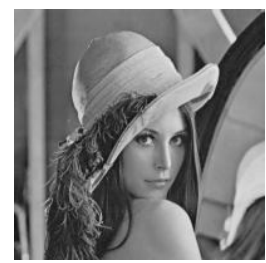

(a)

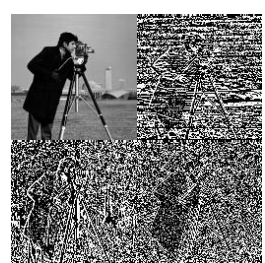

(b)

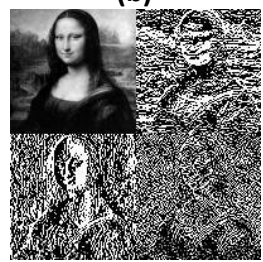

(e)

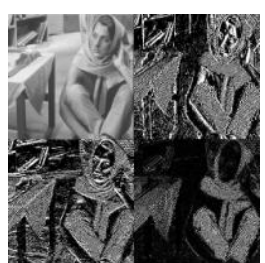

(c)

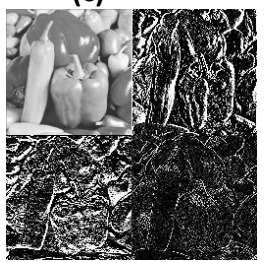

(f)

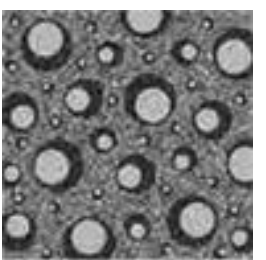

(d)

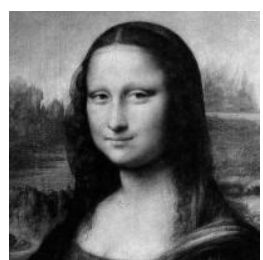

(e)

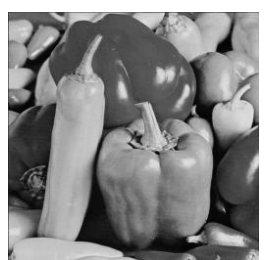

(f)

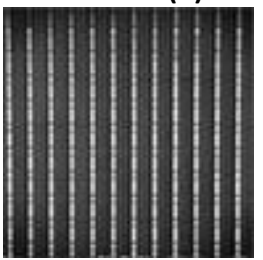

(g)

Figure 7: Image with Watermarking (a) Lena Image (b) Cameraman Image (c) Barbara Image (d) Circle Image (e) Monalisa Image (f) Pepper Image (g) Line Image

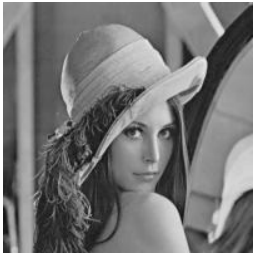

(a)

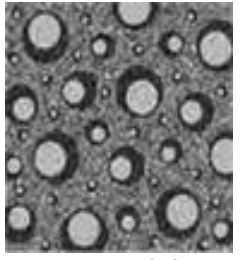

(d)

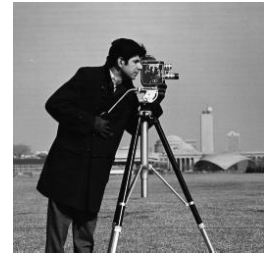

(b)

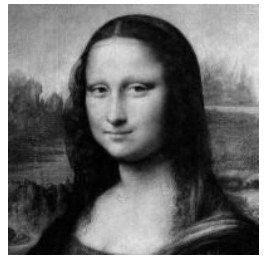

(e)

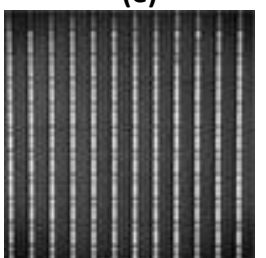

(g)

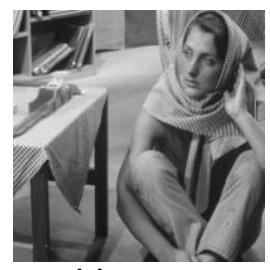

(c)

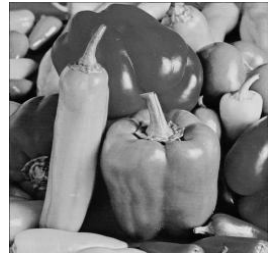

(f)

)


Table 1. Quality Measures for the Considered Images Based on Threshold Maximum Number of Occurences

\begin{tabular}{|l|l|l|l|l|l|l|l|l|}
\hline S No & Image & Size & MSE & RMSE & SNR & RSNR & PSNR & NCC \\
\hline 1 & Lena & $200 \times 200$ & 28.92 & 5.38 & 24214000.00 & 4920.70 & 33.52 & 0.99 \\
\hline 2 & CameraMan & $256 \times 256$ & 52.42 & 7.24 & 22442000.00 & 4737.30 & 30.94 & 0.99 \\
\hline 3 & Barbara & $512 \times 512$ & 53.03 & 7.28 & 8265100.00 & 9091.30 & 30.88 & 0.99 \\
\hline 4 & Circle & $256 \times 256$ & 3.72 & 1.93 & 89906000.00 & 9481.90 & 42.43 & 0.99 \\
\hline 5 & Monalisa & $200 \times 200$ & 20.00 & 4.49 & 28526000.00 & 5341.00 & 35.07 & 0.99 \\
\hline 6 & Pepper & $256 \times 256$ & 25.97 & 5.09 & 43756000.00 & 6614.80 & 33.98 & 0.99 \\
\hline 7 & Line & $256 \times 256$ & 30.88 & 5.56 & 18851000.00 & 4341.80 & 33.23 & 0.99 \\
\hline
\end{tabular}

Table 2. Quality Measures for the Considered Images Based on Threshold (Minimum + Maximum /2)

\begin{tabular}{|l|l|l|l|l|l|l|l|l|}
\hline S No & Image & Size & MSE & RMSE & SNR & RSNR & PSNR & NCC \\
\hline 1 & Lena & $200 \times 200$ & 28.71 & 5.36 & 24389000.00 & 4938.50 & 33.55 & 0.99 \\
\hline 2 & CameraMan & $256 \times 256$ & 52.53 & 7.25 & 22395000.00 & 4732.40 & 30.93 & 0.99 \\
\hline 3 & Barbara & $512 \times 512$ & 53.07 & 7.28 & 82599000.00 & 9088.00 & 30.88 & 0.99 \\
\hline 4 & Circle & $256 \times 256$ & 3.75 & 1.94 & 89158000.00 & 9442.40 & 42.39 & 0.99 \\
\hline 5 & Monalisa & $200 \times 200$ & 20.06 & 4.47 & 28714000.00 & 5358.50 & 35.10 & 0.99 \\
\hline 6 & Pepper & $256 \times 256$ & 26.08 & 5.10 & 4356000.00 & 6600.00 & 33.96 & 0.99 \\
\hline 7 & Line & $256 \times 256$ & 30.87 & 5.56 & 18856000.00 & 4342.30 & 33.24 & 0.99 \\
\hline
\end{tabular}

Table 3. Quality Measures for the Considered Images Based on Threshold Mean

\begin{tabular}{|l|l|l|l|l|l|l|l|l|}
\hline S No & Image & Size & MSE & RMSE & SNR & RSNR & PSNR & NCC \\
\hline 1 & Lena & $200 \times 200$ & 28.68 & 5.36 & 24421000.00 & 4941.80 & 33.56 & 0.99 \\
\hline 2 & CameraMan & $256 \times 256$ & 53.38 & 7.31 & 22039000.00 & 4694.50 & 30.86 & 0.99 \\
\hline 3 & Barbara & $512 \times 512$ & 53.07 & 7.28 & 82592000.00 & 9088.00 & 30.88 & 0.99 \\
\hline 4 & Circle & $256 \times 256$ & 3.71 & 1.93 & 90177000.00 & 9496.20 & 42.44 & 0.99 \\
\hline 5 & Monalisa & $200 \times 200$ & 20.19 & 4.49 & 28528000.00 & 5341.20 & 35.07 & 0.99 \\
\hline 6 & Pepper & $256 \times 256$ & 26.54 & 5.15 & 42809000.00 & 6542.90 & 33.89 & 0.99 \\
\hline 7 & Line & $256 \times 256$ & 30.79 & 5.55 & 18903000.00 & 4347.80 & 33.25 & 0.99 \\
\hline
\end{tabular}


Table 4. Quality Measures after attacks for the Considered Images.

\begin{tabular}{|l|l|l|l|l|}
\hline S No & Image & Size & PSNR & NCC \\
\hline 1 & Lena & $200 \times 200$ & 29.26 & 0.82 \\
\hline 2 & CameraMan & $256 \times 256$ & 25.64 & 0.78 \\
\hline 3 & Barbara & $512 \times 512$ & 25.05 & 0.78 \\
\hline 4 & Circle & $256 \times 256$ & 39.95 & 0.92 \\
\hline 5 & Monalisa & $256 \times 256$ & 30.82 & 0.86 \\
\hline 6 & Pepper & $256 \times 256$ & 30.01 & 0.85 \\
\hline 7 & Line & $256 \times 256$ & 30.51 & 0.85 \\
\hline
\end{tabular}

\section{CONCLUSIONS}

The proposed TIBV-DW method guarantees high authentication due to two steps where the first step indicates where to insert and the second step indicates how to insert digital watermark. The quality measures of T1, T2, T3 shows the similar values for all three thresholds considered in the present approach. The lower values of MSE, RMSE and higher values of SNR, RSNR, around $30 \mathrm{db}$ value of PSNR and around 0.78 of NCC for all the images considered indicate high robustness and better quality for all the reconstructed images. The considered images show high perceptual quality. The table 4 clearly indicates the sustainability of the proposed method over various combinations of attacks, instead of a single attack.

\section{ACKNOWLEDGMENTS}

The authors would like to express their cordial thanks to Sri. K.V.V. Satya Narayana Raju, MLC and Chairman for providing facility to work at the advanced labs of Srinivasa Ramanujan Research Forum (SRRF)-GIET and Sri. K. Sasi Kiran Varma, Managing Director, Chaitanya Group of Institutions for providing moral support and encouragement towards research.

\section{REFERENCES}

[1] Ju-YuanHsiao, Ke-FanChan, J.MorrisChang, "Block Based Reversible Data Embedding”, Signal Processing '89 (2009) 556-569

[2] S.MaruthuPerumal, B.VijayaKumar, L.Sumalatha and Dr V Vijayakumar, "Wavelet Based Watermarking Technique using Simple Preprocessing Method", IJCNS Vol1,No 2 Nov 2009 ISSN Online: 2076-9199

[3] Peter Meerwald and Andreas Uhl, , "A Survey of WaveletDomain Watermarking Algorithms," in Proceedings of Society of Photographic Instrumentation Engineers (SPIE), Vol. 4314, pp. 505-516.

[4] G.RoslineNesaKumari, B.VijayaKumar, L.Sumalatha and Dr V V Krishna, "Secure and Robust Digital Wateramrking on Grey level Images"., IJAST Vol 11 Oct 2009 ISSN: 2005-4238
[5] Dr V Vijayakumar, S.MaruthuPerumal and G.Rosline Nesa Kumari, "A novel Method of Digital Watermarking for High Authentication"., Proceedings of the National Conference on Informatics(NCI)-Nellore - A.P.-India2008.

[6] Ravi K Sharma and Steve Decker, "Practical Challenges for Digital Watermarking Applications", EURASIP Journal on Applied Signal Processing 2002. 133-139

[7] S.MaruthuPerumal, L.Sumalatha, Dr V VenkataKrishna, "Digital Image Authentication Based on Threshold Values", Proceedings of the 1st International Conference on Emerging Trends in Signal Processing \& VLSI Design.,Hyderabad-A.P.India- 2010.

[8] Akram M Zeki and Azizah A Manaf, "A Novel Digital Watermarking Technique based on ISB", Proceedings of World Academy of Science, Engineering and Technology, Vol 38, Feb 2009 ISSN 2070-3740.

[9] Zhe-Ming Lu, Chun-He Liu and He Wang, "Image Retrieval and content Integrity Verification Based on Multipurpose Image Watermarking Scheme" International journal of Innovative Computing, Information and Control Vol - 3,Number 3, June 2007.

[10] WANG Pengfei, QIN Feng, WANG Xiaolin, FENG Gui, CAI Canhui, "A Novel Blind Watermarking Scheme Based on Watermark Hopping Technique". ICSP2006 Proceedings.

[11] Soumik Das, Pradosh Bandyopadhyay, Shauvik Paul, Arindam Sinha Ray and Dr. Monalisa Banerjee, "A New Introduction towards Invisible Image Watermarking on Color Images". IEEE International Advance Computing Conference (IACC 2009)

[12] Santi P. Maity , Malay K. Kundu , Seba Maity, "Dual purpose FWT domain spread spectrum image watermarking in real time" Computers and Electrical Engineering 35 (2009) 415-433

[13] Hong-Jie He , Jia-ShuZhang , FanChen “Adjacent-lock based statistical detection method for self-embedding watermarking techniques", Signal Processing 89 (2009) $1557-1566$.

[14] Shumei Wang, Weidong Zhao, Zhicheng Wang, Junwei D, Xialin Han, "A Blind and Self-Adaptive Digital Watermarking Based on Wavelet-packet Decomposition" IEEE 2008 Congress on Image and Signal Processing 244248

[15] Alessandro Basso, Francesco Bergadano, Davide Cavagnino, Victor Pomponiu and Annamaria Vernone, "A Novel Block-based Watermarking Scheme Using the SVD Transform" Algorithms 2009 ISSN 1999-4893.

[16] G.Rosline Nesa Kumari, B.VijayaKumar, Dr D. Rajya Lakshmi, "A Novel Approach of Digital Watermarking on Grey Level Images", Proceedings of the 1st International Conference on Emerging Trends in Signal Processing \& VLSI Design.,Hyderabad-A.P.India- 2010.

[17] B.V. Ramana Reddy, M. Radhika Mani, K.V. Subbaiah, "Texture Classification Method using Wavelet Transforms Based on Gaussian Markov Random Field" International Journal of Signal and Image Processing (Vol.1-2010/Iss.1) 
[18] Daubechies Ingrid “Ten Lectures on Wavelets," Society for Industrial and Applied Mathematics, 1992.

[19] Jaideva C. Goswami and Andrew K. Chan "Fundamentals of Wavelets Theory, Algorithms, and Applications," A wiley- Interscience Publications.

[20] Michael W. Frazier "An Introduction to Wavelets through Linear Algebra," Replika Press Pvt Ltd, Delhi, India, 1999.

[21] Nievergelt Yves "Wavelets Made Easy," R.R. Honnelley and Sons, Harrisonburg, USA, 1999.

[22] Pankaj N. Topiwala "Wavelet Image and Video Compression", Kluwer Academic Publishers, 1998.

[23] Prasad L. and Iyengar S.S. "Wavelet analysis with applications to image processing," CRC press LLC, Florida, USA, 1997.

[24] Rafael C. Gonzalez and Richard E. Woods "Digital Image Processing," 2nd ed., Pearson Education (Singapore) Pte. Ltd, Indian Branch, 2003.

[25] Raghuveer M. Rao and Ajit S. Bopardikar "Wavelet Transforms-Introduction to Theory and Applications," Pearson Education, Published by Dorling Kindersley (India) Pvt. Ltd, 2006.

[26] Soman K.P. and Ramachandran K.I. "Insight into wavelets from theory to practice," Prentice-Hall of India Pvt. Limited, 2004.

[27] Soman K.P. and Ramachandran K.I. "Insight into wavelets from theory to practice," Second Edition, Prentice-Hall of India Pvt. Limited, 2006.

[28] C.C.Chang. T.C. Lu, A difference Expansion oriented data hiding scheme for restoring the original host images,J.Systems Software 79 (May 2006) 1754-1766

[29] J.Tian, Reversible data embedding using a difference expansion, IEEE Trans, circuits systems video Technol. 13(8) (August 2003) 890-896

[30] J.M Barton. Method and Apparatus for embedding authentication information within digital data, U.S patent 5 6469971997.

[31] M.U.Celik, G.Sharma, A.M.Tekalp, E.Saber, Lossless generalized LSB Data embedding, IEEE Trans Process. 14 (2) February 2005)253-266.
[32] C.De Vleeschouwer, J.F.Delaigle, B.Macq, Circular interpretation of bijective transformation in lossless watermarking for media asset management, IEEE Trans Multimedia 5(1) (March 2003) 97-105.

[33] B.Macq, Lossless multiresolution transform for image authenticating watermarking, in :Proceeding of EUSIPCO, Vol. 2000, Tempere Finland, September 2000, pp 533-536.

[34] D.M.Thodi, J.J.Rodriguez, Prediction error based reversible watermarking in proceedings of the international conference on image processing (ICIP'04) vol 3, Singapore, 24-27 October 2004 pp 1549-1552.

\section{AUTHORS PROFILE}

S.MaruthuPerumal received his B.E, in Computer Science and Engineering from Bharathidasan University and M.E. in Computer Science and Engineering from Sathyabama University Chennai. He is having twelve years of teaching experience. At present he is working as an Associate Professor in the Department of IT Godavari Institute of Engineering and Technology, Rajahmundry. He published 7 research publications in various Inter National, National Conferences and Journal. He is an active member of Srinivasa Ramanujan Research Forum (SRRF)-GIET, Rajahmundry. He is Pursuing his Ph.D at Dr MGR University Chennai under the Guidance of Dr.V.VijayaKumar. His research interest includes Image processing, Digital Watermarking, Steganography and Security. $\mathrm{He}$ is a life member of ISCA,IAENG and Institutional Member of CSI.

Vakulabharanam Vijaya Kumar received integrated M.S. Engg, degree from Tashkent Polytechnic Institute (USSR) in 1989. He received his Ph.D. degree in Computer Science from Jawaharlal Nehru Technological University (JNTU) in 1998. He has served the JNT University for 13 years as Assistant Professor and Associate Professor and taught courses for M.Tech students. He has been Dean for Dept of CSE and IT at Godavari Institute of Engineering and Technology since April, 2007.His research interests include Image Processing, Pattern Recognition, Network Security, Steganography, Digital Watermarking, and Image retrieval. He is a life member for CSI, ISTE, IE, IRS, ACS and CS. He has published more than 120 research publications in various National, Inter National conferences, proceedings and Journals. 